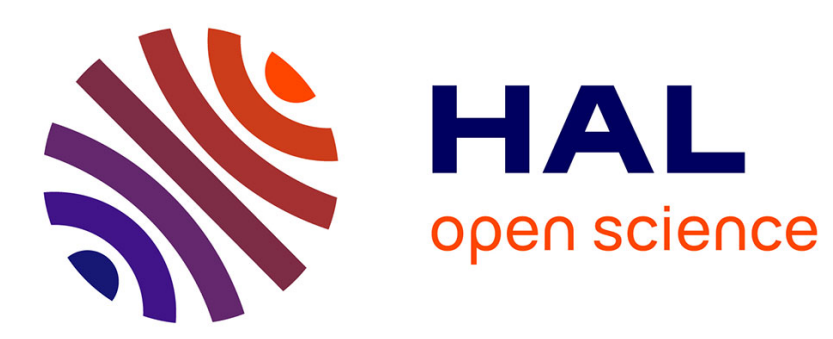

\title{
Coincidence of Condorcet committees
}

Eric Kamwa, Vincent Merlin

\section{To cite this version:}

Eric Kamwa, Vincent Merlin. Coincidence of Condorcet committees. Social Choice and Welfare, 2018, 50 (1), pp.171-189. 10.1007/s00355-017-1079-z . hal-01631176

\section{HAL Id: hal-01631176 https://hal.univ-antilles.fr/hal-01631176}

Submitted on 27 Jun 2019

HAL is a multi-disciplinary open access archive for the deposit and dissemination of scientific research documents, whether they are published or not. The documents may come from teaching and research institutions in France or abroad, or from public or private research centers.
L'archive ouverte pluridisciplinaire HAL, est destinée au dépôt et à la diffusion de documents scientifiques de niveau recherche, publiés ou non, émanant des établissements d'enseignement et de recherche français ou étrangers, des laboratoires publics ou privés. 


\title{
Coincidence of Condorcet Committees
}

\author{
Eric Kamwa* $\quad$ Vincent Merlin ${ }^{\dagger}$
}

\begin{abstract}
The Condorcet Committee à la Gehrlein (CCG) is a fixed-size subset of candidates such that each of its members defeats in a pairwise contest any candidate outside. The Condorcet Committee à la Fishburn (CCF) is a fixedsize subset of candidates that is preferred to all other subsets of the same size by a majority of voters. In general, these two types of Condorcet committees may not always exist. Kaymak and Sanver (2003) studied the conditions under which the CCF exists under a large class of extensions of preferences. We focus here on the most important members of their class, the lexicographic extension of preferences, and we define more precisely, the conditions under which these committees coincide when they exist. Our results depart from the rather optimistic conclusions of Kaymak and Sanver (2003) on the coincidence between the CCG and the CCF. We exhibit profiles for which the CCF is empty while the CCG exists and the preferences are all of lexicographic type. Furthermore, we obtain the same conclusion when we derive preferences on candidates from those on sets of candidates using the separability assumption.
\end{abstract}

JEL Classification Numbers : D70, D71

Keywords : Condorcet, Committees, Preference, Lexicographic extension, Separable preferences.

${ }^{*}$ Université des Antilles, Faculté de Droit et d'Economie de la Martinique and Laboratoire Caribéen de Sciences Sociales LC2S UMR CNRS 8053, F-97275 Schoelcher Cedex. Email: eric.kamwa@univ-antilles.fr $(\bowtie)$.

${ }^{\dagger}$ CNRS and Normandie Univ, France ; UNICAEN, CREM, UMR-CNRS 6211. UFR des Sciences Economiques, Gestion, Géographie et Aménagement du Territoire, Esplanade de la Paix, F14032 Caen. Email: vincent.merlin@unicaen.fr 


\section{Introduction}

In a representative democracy, there are two possible ways to elect a parliament or a committee of representatives. Voters can provide their preferences (rankings) over (1)-the set of the competing candidates (we call this the Candidate approach) or over (2)-the set of the possible committees given the size of the committee to be elected (we call this the Committee approach). With the first (resp. the second) approach, the elected committee will be formed by the candidates (resp. the committee) with the most support given the voting rule. Notice that the first approach prevails in the social choice literature as well as in practice.

According to Coelho (2004), what comes from the debate that saw Dodgson $(1876,1884,1885 a, b)$ in opposition to the Society for Proportional Representation ${ }^{1}$ is that when electing a committee, dissociating voters' preferences on candidates from those they might have on subsets of candidates can lead to dominated committees. Following Condorcet (1785), a candidate (resp. committee) is dominated when there is another candidate (resp. committee) that is preferred to him by a majority of the electorate. The discussion between Dodgson and the Society for Proportional Representation opened the path to two solution concepts when electing fixed-size committees: one for electing undominated committees and the other one for electing undominated candidates. The following questions can be asked: Given a voting situation, is it possible to have a committee that is undominated and that is made of undominated candidates? This question has been tackled in a certain way by Kaymak and Sanver (2003) when the preferences on candidates are extended in a certain way on subsets. One of the main objectives of this paper is to check particular voting situation and investigate how, when it exists, a committee can be simultaneously undominated and made of undominated candidates. To perform this comparison, we will focus on a subgroup of preference extensions included in the Kaymak and Sanver (2003) class, the Leximin and Leximax extensions.

Under the Candidate approach, Gehrlein (1985) proposed the Condorcet Committee à la Gehrlein (CCG) as a way to get a committee made of undominated candidates. The CCG is a fixed-size subset of candidates such that each of its members defeats in a pairwise contest any candidate outside. ${ }^{2}$ To our knowledge, the intuition behind the CCG is due to Good (1971); he was the first to introduce the Condorcet Set as the minimal set of candidates such that every member in this set beats in pairwise majority every candidate outside. As noticed by Good (1971), the

\footnotetext{
${ }^{1}$ See also Black (1958); McLean and Urken (1995).

${ }^{2}$ This definition of the CCG is also known in the literature as the Strong Condorcet Committee; see also Kaymak and Sanver (2003); Ratliff (2003).
} 
Condorcet Set may not always be a proper subset of the set of all candidates. In case of a complete majority cycle, it is made up of the whole set of candidates. In contrast, the Committee approach was defended by Fishburn (1981) who proposed the Condorcet Committee à la Fishburn (CCF) as a fixed-size subset of candidates that is majority preferred to all the other subsets of the same size.

Hill (1988), Kaymak and Sanver (2003) focused on the compatibility between the CCG and the CCF. They concluded that the CCG will be well suited only if the elected committee is a first list of candidates that will be submitted afterwards to deliberation in order to determine a final winner from this list. Nonetheless, if the members of the elected committee must accurately reflect the electorate, the CCF will be more suited. Hence, by selecting the CCF when it exists, we are sure that we won't get a dominated committee. Similarly, selecting the CCG when it exists will prevent the selection of dominated candidates. Ideally, we would like a set to be both a CCG and a CCF at the same time. In such a case, the legitimacy of the elected committee could not be contested on the basis of any majority criterion.

With the Candidate approach, voters only provide their rankings over candidates and there is no means to know how they rank subsets of candidates. Many studies have tried to construct mechanisms to derive voters' preferences on subsets of candidates from those on candidates. Packard (1979), Nitzan and Pattanaik (1984), Barberà et al. (1984), Barberà et al. (2001) among others, studied the question of how rankings on individual candidates can be extended to those on committees. We will follow this approach by going from preferences on candidates onto those on subsets of candidates. Although there are various techniques for preferences extensions, we will mainly focus here on two lexicographical ones.

Kaymak and Sanver (2003) proved that a committee is a CCF if and only if there exist extensions of preferences satisfying certain conditions that ensure it will also be undominated in a certain way. As we will see, their main condition is a kind of majority condition but it is quite different from the one classically used for the definition of the CCG. Also, the class of extensions proposed by Kaymak and Sanver (2003) contains the Leximax and the Leximin extensions as specific cases. However, a weakness with their results comes from the fact that, to obtain the existence of a CCF, different extensions within their class may have to be used with different profiles. The objective of our paper will be to revisit their results when all the voters systematically use the same extension at each profile. In particular we will show that when the voters are all consistently Leximax (resp. Leximin): 1) a $C C G$ may exist while there is no CCF and 2) if there is no CCG there is also no CCF by extension. We will also discuss the links between the CCG and the majority domination condition proposed by Kaymak and Sanver (2003). 
Symmetrically, Benoit and Kornhauser (1991, 1994, 1999) pointed out that the Candidate approach has to be evaluated in terms of the Committee approach as preferences over committees are fundamental when electing committees. Using the separability condition, they described a natural way of getting preferences on candidates when one cares about the committee as a whole. We will show that we obtain the same conclusion as under the lexicographic approach when we derive preferences on candidates from those on sets of candidates using the separability assumption.

The rest of the paper is structured as follows: Section 2 is devoted to basic notations and definitions. Section 3 presents the theoretical underpinnings of preference extensions; in this section, we analyze the majority relation when preferences are extended. In Section 4, some general results are provided on the relationships between the CCG and the CCF with lexicographic extension. We discuss the limits of our approach in Section 5. In Section 6 we deal with one natural way of deriving voters' preferences on candidates from those on committees. Section 7 concludes.

\section{Notation and definitions}

Let $N$ be the set of $n$ voters (with $n \geq 3$ ) and $A$ the set of $m$ candidates (with $m \geq 3$ ). The aim is to elect a committee of size $g$ with $2 \leq g \leq m-1$. A committee is a fixed-size subset of $A$. We denote by $\mathcal{A}^{g}$ the set of all subsets of $A$ of size $g$. With $m$ candidates, there are exactly $m_{g}=\frac{m !}{g !(m-g) !}$ committees of size $g$ and $m_{g}$ ! possible strict rankings on these committees. Before going further, we need to define voters' preferences on $A$ and on $\mathcal{A}^{g}$.

\section{$2.1 \quad$ Preferences}

We assume that voters have strict rankings on candidates and committees. The binary relation $R$ over $A$ is a subset of the cartesian product $A \times A$. For $a, b \in A$, if $(a, b) \in R$, we note $a R b$ to say that " $a$ is at least good as $b$ ". $\neg a R b$ is the negation of $a R b$. If we have $a R b$ and $\neg b R a$, we will say that " $a$ is better or strictly preferred to $b$ ". In this case, we write $a P b$ with $P$ the asymmetric component of $R$. The symmetric component of $R, I$, is defined by $a I b$ for an indifference between $a$ and $b$ i.e $a R b$ and $b R a$. The preference profile $\pi=\left(P_{1}, P_{2}, \ldots, P_{i}, \ldots, P_{n}\right)$ gives all the linear orders ${ }^{3}$ of

\footnotetext{
${ }^{3} \mathrm{~A}$ linear order is a binary relation that is transitive, complete and antisymmetric. The binary relation $R$ on $A$ is transitive if for $a, b, c \in A$, if $a R b$ and $b R c$ then $a R c$. $R$ is antisymmetric if for all for $a \neq b, a R b \Rightarrow \neg b R a$; if we have $a R b$ and $b R a$, then $a=b . R$ is complete if and only if for all $a, b \in A$, we have $a R b$ or $b R a$.
} 
all the $n$ voters on $A$ where $P_{i}$ is the strict ranking of a given voter $i$. The set of all the preference profiles of size $n$ on $A$ is denoted by $P(A)^{n}$.

The binary relation $R^{*}$ on $\mathcal{A}^{g}$ is a subset of the cartesian product $\mathcal{A}^{g} \times \mathcal{A}^{g}$. For $C_{1}, C_{2} \in \mathcal{A}^{g}, C_{1} R^{*} C_{2}$ means that " $C_{1}$ is at least good as $C_{2}$ ". In a similar way, $R^{*}, I^{*}$ and $P^{*}$ are defined on $\mathcal{A}^{g}$. The profile $\pi^{*}=\left(P_{1}^{*}, P_{2}^{*}, \ldots P_{i}^{*}, \ldots, P_{n}^{*}\right)$ gives the linear orders on $\mathcal{A}^{g}$. We denote by $P^{*}\left(\mathcal{A}^{g}\right)^{n}$ the set of all preference profiles of size $n$ on $\mathcal{A}^{g}$. Section 3 will give theoretical tools we use to deduce voters' preferences on committees from those on candidates, in a consistent way.

\subsection{The Condorcet committees}

We need first to define majority relations respectively on $A$ and on $\mathcal{A}^{g}$. For $a, b \in A$, we denote by $n_{a b}(\pi)$ the number of voters who prefer $a$ to $b$ in the profile $\pi$. For two committees $C, C^{\prime} \in \mathcal{A}^{g}, n_{C C^{\prime}}\left(\pi^{*}\right)$ is the number of voters who rank committee $C$ before $C^{\prime}$ in the profile $\pi^{*}$. When there will be no risk of confusion, we will simply write $n_{a b}$ instead of $n_{a b}(\pi)$ and $n_{C C^{\prime}}$ instead of $n_{C C^{\prime}}\left(\pi^{*}\right)$.

Definition 1. For $a, b \in A, a$ is majority preferred to $b$ in the profile $\pi$ if $n_{a b}>n_{b a}$. We denote it by $a M(\pi) b$ or $a M b$ for simplicity.

Definition 2. For $C, C^{\prime} \in \mathcal{A}^{g}, C$ is majority preferred to $C^{\prime}$ in the profile $\pi^{*}$ if $n_{C C^{\prime}}>n_{C^{\prime} C}$. We denote it by $C M^{*}\left(\pi^{*}\right) C^{\prime}$ or simply $C M^{*} C^{\prime}$.

The Condorcet committee defined by Fishburn (1981) is deduced from voters' preferences on committees. On the contrary, the definition given by Gehrlein (1985) is built upon pairwise majority relationships among candidates. Formally, we have the following definitions.

Definition 3. (Condorcet Committee à la Gehrlein (CCG)). Consider a profile $\pi \in$ $P(A)^{n}$. A committee $C \in \mathcal{A}^{g}$ is the CCG if and only if $\forall x \in C$, we have $x M y$ for all $y \in A \backslash C$.

Our analysis is also valid for what is known as the weak version of the CCG: a fixed-size set of candidates such that none of its members is defeated in pairwise comparisons by any outside contenders (see Barberà and Coelho (2008); Kamwa $(2017 \mathrm{a}, \mathrm{b}))$.

Definition 4. (Condorcet Committee à la Fishburn (CCF)). Consider a profile $\pi^{*} \in$ $P^{*}\left(\mathcal{A}^{g}\right)^{n}$. A committee $C \in \mathcal{A}^{g}$ is the CCF if and only if $\forall C^{\prime} \in \mathcal{A}^{g} \backslash C, C M^{*} C^{\prime}$. 
To determine the CCF, we need to know how the voters rank all the committees. In reality, it could be difficult for a voter to rank all the candidates when their number is huge. However, the situation is worse when we operate on committees. Therefore, the Committee approach is not easy to implement. Maybe it is for this reason that Gehrlein (1985) proposed his own definition of the Condorcet committee. We do not want to open a debate about the advantages of one concept over the other. The conclusions of Hill (1988), Kaymak and Sanver (2003) give some arguments on such a matter.

Given a profile in $P^{*}\left(\mathcal{A}^{g}\right)^{n}$, the CCF does not always exist (Fishburn, 1981); the same result holds for the CCG given a profile in $P(A)^{n}$ (Gehrlein, 1985). There is no guarantee that given a unique CCG, no other committee is majority preferred to it via $M^{*}$. To know more about this, we must have, as well as, the rankings of voters on candidates, their rankings on committees in $\mathcal{A}^{g}$. The next section provides the tools to achieve such a goal.

\section{From preferences on candidates to preferences on committees}

Consider $x, y$ in $A$ and $\mathcal{A}$ the set of all the nonempty subsets of $A$. If we have $x P y$, what can we say about the ranking between the subsets $\{x\},\{y\}$ and $\{x, y\}$ ? There is a large range of works in the social choice literature that proposes answers to this issue ${ }^{4}$. Barberà et al. (2001) give a remarkable survey of this literature. They describe various methods for preference extensions and present numerous properties. In this paper, only two methods will be considered at first: the Leximax extension and the Leximin extension. Before giving a formal definition of both methods, let us present some properties that an extension method should satisfy.

Recall that in Section 2, we have used $R^{*}, P^{*}, I^{*}$ as binary relations on $\mathcal{A}^{g}$ for preferences over committees. To avoid too much notation in this section and to facilitate the comprehension of our work, this notation will be applied to all subsets of $A$ whatever their size is.

\subsection{The extension rule}

An extension rule defines a way to go from preferences on elements of a set to those on its subsets.

\footnotetext{
${ }^{4}$ Without being exhaustive, we can list Arrow and Hurwicz (1972), Packard (1979), Nitzan and Pattanaik (1984), Pattanaik and Peleg (1984), Barberà et al. (1984), Pérez et al. (2012).
} 
Definition 5. (The extension rule). An extension of $R$ to $R^{*}$ is such that for all $x, y \in A,\{x\} R^{*}\{y\} \Leftrightarrow x R y$.

Definition 6. (The weak extension rule). An extension of $P$ to $P^{*}$ is such that for all $x, y \in A,\{x\} P^{*}\{y\} \Leftrightarrow x P y$.

Barberà et al. (2001) review numerous properties of the extension rules ${ }^{5}$. The Dominance property requires that adding in $X \subset \mathcal{A}$ an element that is better (worse) than all the elements of $X$, leads to a better (worse) set than $X$.

Property 1. (Dominance) $\forall X \in \mathcal{A}$ and $\forall x \in A$,

(i) $[x P y, \forall y \in X] \Rightarrow X \cup\{x\} P^{*} X$;

(ii) $[y P x, \forall y \in X] \Rightarrow X P^{*} X \cup\{x\}$.

The weak version of the property of dominance, Simple Dominance, is applied only for extensions implying singletons.

Property 2. (Simple Dominance) $\forall x, y \in A$,

$$
x P y \Rightarrow\left[\{x\} P^{*}\{x, y\} \text { and }\{x, y\} P^{*}\{y\}\right] .
$$

According to the property of Strict Independence, when a subset is strictly preferred to another subset, adding the same element in both subsets does not reverse the strict ranking between these subsets.

Property 3. (Strict Independence) $\forall X, Y \in \mathcal{A}$ and $\forall x \in A \backslash(X \cup Y)$,

$$
X P^{*} Y \Rightarrow X \cup\{x\} P^{*} Y \cup\{x\} .
$$

By Strict Extended Independence, adding the same subset of elements to two others subsets preserves the strict relation.

Property 4. (Strict Extended Independence) $\forall X, Y \in \mathcal{A}$ and $\forall Z \subseteq A \backslash(X \cup Y)$,

$$
X P^{*} Y \Rightarrow\{X \cup Z\} P^{*}\{Y \cup Z\}
$$

\footnotetext{
${ }^{5}$ As we only consider strict preferences in this paper, we will omit to present the versions of the properties that deal with weak preferences.
} 
The property of Disjoint Independence is similar to the previous two properties with the particularity that it only involves disjoint sets. According to this property, adding the same element to two disjoint sets does not reverse the strict preference relation between these sets.

Property 5. (Disjoint Independence) $\forall X, Y \in \mathcal{A}$ such that $X \cap Y=\emptyset$ and $\forall x \in$ $A \backslash(X \cup Y)$,

$$
X P^{*} Y \Rightarrow X \cup\{x\} P^{*} Y \cup\{x\}
$$

Now, we can focus on the extension techniques used in this paper.

\subsection{The Leximax and the Leximin extensions}

The Leximax extension ranks or compares subsets of candidates according to their best elements. If two subsets have the same best element, the ranking will depend upon the second-best element and so on. At some point, for $X \subseteq Y$, if $X$ and $Y$ are still equivalent according to the Leximax while there is no remaining alternative left in $X$ for further comparison, $X$ is declared better than $Y$.

Definition 7. (Leximax Extension) Consider $X, Y \in \mathcal{A}, X \neq Y$ and $P \in P(A)$ such that $X=\left\{x_{1}, x_{2}, \ldots, x_{h}\right\}, Y=\left\{y_{1}, y_{2}, \ldots, y_{k}\right\}, x_{1} P x_{2} P \ldots P x_{h}, y_{1} P y_{2} P \ldots P y_{k}$ and $h \leq k$. We write $Y \bar{P}^{*} X$ to say $Y$ is preferred to $X$ by Leximax extension of $P$, if there is a $j(1 \leq j<k)$ such that $x_{1}=y_{1}, x_{2}=y_{2}, \ldots$, and $y_{j} P x_{j}$. Otherwise, $X \bar{P}^{*} P$.

Example 1. Consider $A=\{a, b, c\}$ and the preference $a P b P c$. The Leximax ranking on subsets of $A$ associated to $a P b P c$ is

$$
\{a\} \bar{P}^{*}\{a, b\} \bar{P}^{*}\{a, b, c\} \bar{P}^{*}\{a, c\} \bar{P}^{*}\{b\} \bar{P}^{*}\{b, c\} \bar{P}^{*}\{c\}
$$

The Leximin extension is dual of the Leximax. It compares subsets by their worst elements. If two subsets have the same worst element, the ranking depends upon the next worst elements and so on. At some point, for $X \subseteq Y$, if $X$ and $Y$ are still equivalent according to the Leximin while there is no remaining alternative left in $X$ for further comparison, $Y$ is declared better than $X$.

Definition 8. (Leximin Extension). Consider $X, Y \in \mathcal{A}, X \neq Y$ and $P \in P(A)$ such that $X=\left\{x_{1}, x_{2}, \ldots, x_{h}\right\}, Y=\left\{y_{1}, y_{2}, \ldots, y_{k}\right\}, x_{1} P x_{2} P \ldots P x_{h}, y_{1} P y_{2} P \ldots P y_{k}$ and $h \leq k$. We write $X \underline{P}^{*} Y$ to say that $X$ is preferred to $Y$ by Leximin extension of $P$, if there is a $j(0 \leq j<h)$ such that $x_{h}=y_{k}, x_{h-1}=y_{k-1}, \ldots$, and $x_{h-j} P y_{k-j}$. Otherwise, $Y \underline{P}^{*} X$. 
Example 2. Consider $A=\{a, b, c\}$ and the preference $a P b P c$. The Leximin ranking on subsets of $A$ associated to aPbPc is

$$
\{a\} \underline{P}^{*}\{a, b\} \underline{P}^{*}\{b\} \underline{P}^{*}\{a, c\} \underline{P}^{*}\{a, b, c\} \underline{P}^{*}\{b, c\} \underline{P}^{*}\{c\}
$$

Note that all these extension techniques meet the five properties defined above (see Barberà et al. (2001)). Examples 1 and 2 illustrate that for the same ranking on candidates, the Leximax and Leximin extensions differ. In what follows, when there will be no ambiguity in preferences extension between the notations $\bar{P}^{*}$ and $\underline{P}^{*}$, we will simply use the notation $P^{*}$. Nonetheless, notice that the Leximax and Leximin extensions are weak extensions in the sense of Definition 6.

Proposition 1 tells us that the strict order obtained by extension between two subsets that only differ from each other by one element implies the strict order on the two candidates by which they differ and vice-versa.

Proposition 1. Let $x, y \in A$ and $Z \in \mathcal{A}$ such that $Z \subseteq A \backslash\{x, y\}, X=\{x\} \cup Z$ and $Y=\{y\} \cup Z$. By Leximax or Leximin extension,

$$
x P y \Leftrightarrow X P^{*} Y
$$

Proof. Consider $x, y \in A$ and $X, Y, Z \in \mathcal{A}$ such that $Z \subseteq A \backslash\{x, y\}, X=\{x\} \cup Z$ and $Y=\{y\} \cup Z$.

1. By Definition $6,\{x\} P^{*}\{y\} \Leftrightarrow x P y$. By Property 4, we have $\{x\} P^{*}\{y\} \Rightarrow$ $\{x\} \cup Z P^{*}\{y\} \cup Z$; so, $\{x\} P^{*}\{y\} \Rightarrow X P^{*} Y$. It follows that $x P y \Rightarrow X P^{*} Y$.

2. Since $X \cap Y=Z$, by Definition 7 , we have $X P^{*} Y$ by Leximax or Leximin extension only if $x P y$. Thus, $X P^{*} Y \Rightarrow x P y$.

The property stated in Equation 3.1 is called Axiom 2 in Kaymak and Sanver (2003), and as we will show later, it plays an important role in their results.

All the results we are going to state in the sequel hold for both the Leximax extension and the Leximin extension. So, in such a case, we will simply use the term "lexicographic extension" to refer to any of these methods.

Proposition 2. $\forall X, Y \in \mathcal{A}$ such that $X \cap Y=\emptyset$ and $|X|=|Y|$. If $P^{*}$ is a lexicographic extension,

$$
[\forall x \in X, \forall y \in Y, x P y] \Rightarrow X P^{*} Y
$$


The proof of Proposition 2 is omitted since it exactly follows Definitions 7 and 8 . According to Proposition 2, given two disjoint sets of same size, if every element of one set is strictly preferred to every element of the other set, the first set is strictly preferred to the latter.

The property stated in Equation 3.2 is called Axiom 1 by Kaymak and Sanver (2003), and it will also be an important ingredient for them when stating their results.

\subsection{The majority extension}

As we are here going to extend the majority relations to committees, we will use the notation $\bar{M}^{*}$ for the Leximax and $\underline{M}^{*}$ for the Leximin. When there will be no ambiguity, we will simply use the notation $M^{*}$ for both the the Leximax and the Leximin. Moreover, in order to simplify the proofs, we will also use the notation $M^{*}$ when the reasoning is exactly the same for $\bar{M}^{*}$ and $\underline{M}^{*}$.

The property of Strict Extended Independence is at the source of all the extended majority relations we are going to define.

Proposition 3. For all $x, y \in A, x M y \Leftrightarrow\{x\} M^{*}\{y\}$.

Proof.

$$
\begin{aligned}
x M y & \Leftrightarrow \sharp\left\{i: x P_{i} y\right\}>\sharp\left\{i: y P_{i} x\right\} \\
& \Leftrightarrow \sharp\left\{i:\{x\} P_{i}^{*}\{y\}\right\}>\sharp\left\{i:\{y\} P_{i}^{*}\{x\}\right\} \text { by the weak extension rule } \\
& \Leftrightarrow\{x\} M^{*}\{y\}
\end{aligned}
$$

Thus, $x M y \Leftrightarrow\{x\} M^{*}\{y\}$.

Proposition 3 is simply a direct application of the definition of the weak extension rule (see Definition 6). According to Proposition 3, the majority relation between two candidates is the same between the singletons made by these candidates. Definition 6 and Property 4 allow us to extend the majority relations to subsets of more than one element.

Proposition 4. $\forall x, y \in A$ and $\forall Z \subseteq A \backslash\{x, y\}$,

$$
\{x\} M^{*}\{y\} \Leftrightarrow\{x\} \cup Z M^{*}\{y\} \cup Z .
$$

Proof. Consider $x, y \in A$ and $Z \subseteq A \backslash\{x, y\}$ such that $\{x\} M^{*}\{y\}$. By Proposition 3 , we know that $x M y \Leftrightarrow\{x\} M^{*}\{y\}$. By Definition 6, $N_{x y}=\{i \in N: x P y\}=$ $N_{\{x\}\{y\}}=\left\{i \in N:\{x\} P^{*}\{y\}\right\}$. 
$(\Rightarrow)$ By Property 4 , if for a given voter $i$, we have $\{x\} P_{i}^{*}\{y\}$, we also have $\{x\} \cup$ $Z P_{i}^{*}\{y\} \cup Z$ for this voter no matter what $Z$ is. So, the number of voters for whom $\{x\} P^{*}\{y\}$ is the same as the number of voters for $\{x\} \cup Z P^{*}\{y\} \cup Z$. Therefore, if $\{x\} M^{*}\{y\}$, then $\{x\} \cup Z M^{*}\{y\} \cup Z$.

$(\Leftarrow)$ By Proposition 1, $\{x\} \cup Z P^{*}\{y\} \cup Z \Leftrightarrow\{x\} P *\{y\}$ and it follows that $\{x\} \cup$ $Z M^{*}\{y\} \cup Z \Leftrightarrow\{x\} M^{*}\{y\}$.

The proof of Proposition 4 comes from the juxtaposition of Property 4 and Proposition 1. By Proposition 4, adding the same subset to two different singletons does not reverse the majority relation between these singletons.

Proposition 4 only deals with subsets that differ by one element. What can we say about the majority relation between subsets when they differ by more than one element? Consider $X, Y \in \mathcal{A}$ such that $X \cap Y=\emptyset$. If $\forall x \in X, \forall y \in Y, x M y$, nothing can be said a priori about the majority relation between $X$ and $Y$. The following example illustrates this point.

Example 3. $A=\{a, b, c, d\}$ and we have three voters with the following strict rankings:

$$
1: a P_{1} b P_{1} c P_{1} d, \quad 2: d P_{2} a P_{2} b P_{2} c \text { and } 3: c P_{3} a P_{3} b P_{3} d
$$

The Leximax extended preferences of these voters on two-member committees are as follows:

$$
\begin{array}{lll}
1 & : & (a, b) P_{1}^{*}(a, c) P_{1}^{*}(a, d) P_{1}^{*}(b, c) P_{1}^{*}(b, d) P_{1}^{*}(c, d) \\
2 & : & (a, d) P_{2}^{*}(b, d) P_{2}^{*}(c, d) P_{2}^{*}(a, b) P_{2}^{*}(a, c) P_{2}^{*}(b, c) \\
3 & : & (a, c) P_{3}^{*}(b, c) P_{3}^{*}(c, d) P_{3}^{*}(a, b) P_{3}^{*}(b, d) P_{3}^{*}(a, d)
\end{array}
$$

The reader can check that $(a, b)$ is the $C C G(a M c, a M d, b M c$ and $b M d)$, while $(c, d) \bar{M}^{*}(a, b)$.

Remark 1. We have stated that Propositions 3 and 4 hold for $M^{*}$ when all the individuals are of Leximax type or are all of Leximin type. But indeed, these results also hold for any population which contains a mixture of Leximax voters and Leximin voters. This is due to the fact that, for the proof, we only need Definition 6 and Property 4, which are met by both extensions. 


\section{The Condorcet committee coincidence}

Our aim in this section is to highlight the connections that can exist between the set of the CCG and that of the CCF by lexicographic extension when they both exist. Do a CCG and a CCF always coincide when they exist? A partial answer is given by Kaymak and Sanver (2003) via the notion of a consistency map. They define a consistency map $\kappa$ as a correspondence from $P(A)^{n}$, the set of profiles on alternatives, into $P^{*}\left(\mathcal{A}^{g}\right)^{n}$ the set of profiles on subsets. To each $\pi \in P(A)^{n}$, they associate the set $\kappa(\pi)$ which is the set of profiles on subsets where Axiom 1 and Axiom 2 are satisfied. This notion guarantees some consistency between the individual's behaviors in the contexts of candidate selection and set selection, but it remains quite loose. They do not ask an individual to have the same rationality from profile to profile, nor two different individuals to use the same extension rule, as long as Axioms 1 and 2 are satisfied.

Next, they define a rather strong version of domination. Given a specific $g$, take any $X, Y \in \mathcal{A}^{g}$. Without loss of generality, let $X=\left\{x_{1}, x_{2}, \ldots x_{g}\right\}$ (resp. $\left.Y=\left\{y_{1}, y_{2}, \ldots, y_{g}\right\}\right)$ such that $x_{j} R_{i} x_{j+1}\left(\operatorname{resp} y_{j} R_{i} y_{j+1}\right)$. For individual $i$ and the relation ${ }^{6} R_{i}$, the set $X$ dominates the set $Y$, denoted by $X D_{i} Y$, if and and only if $x_{j} R_{i} y_{j}$ for all $j=1 \ldots g$. In words, $X$ dominates $Y$ if the $j$ th best element of $X$ is always preferred to the $j$ th best element of $Y$ by individual $i$. At the profile level, they will say that $X$ dominates $Y$, denoted by $X D(\pi) Y$ if and only if:

$$
\#\left\{i \in N \mid X D_{i} Y\right\}>\frac{n}{2}
$$

We can now import their Theorem 5.2 into our context:

Theorem 1. (Kaymak and Sanver (2003)). Let $\kappa$ be a consistency map defined by Axiom 2 only. The set $X$ is a CCF of size $k$ for some profile $\pi^{*}$ in $\kappa(\pi)$ if and only if there is no $Y \in \mathcal{A}^{g}$ such that $Y D(\pi) X$.

Proof. See Kaymak and Sanver (2003, p.491).

Nonetheless, notice that this theorem does not apply to our context for one main reason. We are concerned in this paper with the Leximax (resp. Leximin) cases: all the individuals have the same rational behavior both within a profile and across profiles. We need to present a specific analysis, which has not been presented in Kaymak and Sanver (2003).

\footnotetext{
${ }^{6}$ Recall that $a R_{i} b$ means that individual $i$ finds $a$ at least as good as $b$.
} 
Before stating our results, let us motivate our approach, with Example 3 where we have $a M b, a M c, a M d, b M c, b M d$ and $c M d$. Thus for $g=2, C C G=(a, b)$. The leximax-extended preferences of these voters on two-member committees lead to

$$
(a, b) \bar{M}^{*}(a, c) \bar{M}^{*}(a, d) \bar{M}^{*}(b, c) \bar{M}^{*}(b, d) \bar{M}^{*}(c, d) \bar{M}^{*}(a, b)
$$

We have a cycle among the committees, viz. there is no CCF. So, given that there is a CCG, this committee could be a dominated one by leximax-extended preferences. Applying the Kaymak and Sanver (2003) domination relation as described above to Example 3, it follows that,

$$
\begin{aligned}
& \left\{i \in N \mid(a, b) D_{i}(a, c)\right\}=\{i \in N \mid(a, b) D(\pi)(b, c)\}=\{1,2\} \\
& \left\{i \in N \mid(a, b) D_{i}(a, d)\right\}=\{i \in N \mid(a, b) D(\pi)(b, d)\}=\{1,3\} \\
& \left\{i \in N \mid(a, b) D_{i}(c, d)\right\}=\{1\} \\
& \left\{i \in N \mid(c, d) D_{i}(a, b)\right\}=\emptyset
\end{aligned}
$$

Hence, committee $(a, b)$ is undominated in Kaymak and Sanver (2003)'s terminology; but it is not a CCF under the more restrictive leximax extension we use.

Similar conclusions hold for the leximin-extended preferences, as is shown in Example 4.

Example 4. For $A=\{a, b, c, d\}$, we have three voters with the following strict preferences $^{7}$ :

$$
1: a P_{1} b P_{1} c P_{1} d, \quad 2: a P_{2} c P_{2} d P_{2} b, \quad 3: b P_{3} c P_{3} d P_{3} a
$$

The reader can check that we have for $g=2, C C G=(a, b)$ and that by the Leximin extension, $(c, d) \underline{M}^{*}(a, b)$.

In this profile, the Kaymak and Sanver (2003) domination relation will lead to:

$$
\begin{aligned}
\left\{i \in N \mid(a, b) D_{i}(a, c)\right\} & =\{i \in N \mid(a, b) D(\pi)(a, d)\}=\{1,3\} \\
\left\{i \in N \mid(a, b) D_{i}(b, c)\right\} & =\{i \in N \mid(a, b) D(\pi)(b, d)\}=\{1,2\} \\
\left\{i \in N \mid(a, b) D_{i}(c, d)\right\} & =\{1\} \\
\left\{i \in N \mid(c, d) D_{i}(a, b)\right\} & =\emptyset
\end{aligned}
$$

So, $(a, b)$ is undominated; no matter that $(a, b)$ is the $C C G$ and is undominated, it is not a CCF under the leximin extension.

\footnotetext{
${ }^{7}$ We thank an anonymous referee for suggesting this profile with three voters.
} 
So, by Examples 3 and 4, we see that one can have a CCG without a CCF for each of our lexicographic extensions. Similarly, a set may be undominated without being a CCF. Nonetheless, in Theorem 2, we show that if there is a CCF by extension, this committee is also a CCG.

Theorem 2. Consider a preference profile with $m \geq 3$ candidates, assume that all the voters share the same lexicographic extension. If by lexicographic extension there is a $C C F$, it is certainly a $C C G$.

Proof. Suppose for a given voting situation with $m \geq 3$ and $g \leq m-1$ that by lexicographical extension the set of the $\mathrm{CCF}$ denoted by $\mathrm{CCF}_{g}$ is not empty: there is a committee $\tilde{C} \in \mathrm{CCF}_{g}$. We denote by $\mathrm{CCG}_{g}$ the set of the CCG. Let us assume that $\tilde{C} \notin \mathrm{CCG}_{g}$. This means that there is a committee $C$ such that with $a \in C(a \notin \tilde{C})$ and $b \in \tilde{C}(b \notin C)$ we have $a M b$. If we define $Z=C \cap \tilde{C}$ such that $C=Z \cup\{a\}$ such that and $\tilde{C}=Z \cup\{b\}$, it follows that

$$
\begin{aligned}
a M b & \Leftrightarrow\{a\} M^{*}\{b\} \quad \text { by Proposition } 3 \\
& \Leftrightarrow\{a\} \cup Z M^{*}\{b\} \cup Z \text { by Proposition } 4 \\
& \Leftrightarrow C M^{*} \tilde{C} \quad \text { Contradiction }
\end{aligned}
$$

Thus, if there a CCF by lexicographic extension, this committee is also a CCG.

As we have learnt that there can exist a CCG although this committee is not a CCF (Examples 3 and 4), it then clearly follows that a CCF is certainly a CCG while the reverse is not always true. As there may well exist a CCG without this being the case for the CCF, what can be said when there is no CCG? As a simple corollary of Theorem 2, the answer is as follows.

Theorem 3. Given a preference profile on $m \geq 3$ candidates, assume that all the voters share the same lexicographic extension. If there is no CCG of size $g$, there is also no CCF by lexicographic extension.

Thus, when there is no CCG of size $g$, there is also no CCF by lexicographic extension.

Remark 2. We have stated that Theorems 2 and 3 hold for $M^{*}$ when all the individuals are of Leximax type or are all of Leximin type. But indeed, these theorems also hold for any population which contains a mixture of Leximax voters and Leximin voters. This is due to the fact that, for the proof, we only need Definition 6, Propositions 3 and 4 , which are met by both extensions. 
We can now examine more closely the relationships between the CCF, the CCG and the Kaymak and Sanver (2003) domination condition. As the CCF is always a CCG, the CCG is also undominated when they coincide. It remains to study the case where a CCG exists, while this is not the case for the CCF. The following example first shows that a CCG could be dominated in this case.

Example 5. Consider a voting profile with seven voters with the following ranking on $A=\{a, b, c, d\}$ :

$$
\begin{array}{llll}
1: a P_{1} b P_{1} c P_{1} d, & 2: a P_{2} b P_{2} d P_{2} c, & 3: b P_{3} a P_{3} c P_{3} d, & 4: c P_{4} a P_{4} d P_{4} b \\
5: c P_{5} b P_{5} d P_{5} a, & 6: d P_{6} a P_{6} c P_{6} b, & 7: d P_{7} b P_{7} c P_{7} a
\end{array}
$$

For $g=2$, the reader can check that $C C G=(a, b)$ and that this $C C G$ is not a $C C F$ by lexicographic extensions since $(c, d) M^{*}(a, b)$. Also, we can check that $(c, d) D(\pi)(a, b)$ since,

$$
\begin{array}{ll}
\sharp\left\{i \in N \mid(a, b) D_{i}(c, d)\right\} & =\sharp\{1,2,3\}=3 \\
\sharp\left\{i \in N \mid(c, d) D_{i}(a, b)\right\} & =\sharp\{4,5,6,7\}=4
\end{array}
$$

It follows that a CCG could be a dominated committee. We conclude that an undominated CCG could exist without a CCF (see also Examples 3 and 4) but when there is a CCF by lexicographic extension of preferences this set is also an undominated $C C G$ in the sense of Kaymak and Sanver (2003).

\section{The limits of the lexicographic extension: other extension rules}

As in Kaymak and Sanver (2003), we assumed that the voters have a strong form of rationality when extending their preferences on candidates to preferences on subsets. In this section, we want discuss the limits of this approach.

It is clear that if an elected committee has to solve specific tasks requiring different skills, a good committee then will be one where the candidate skills are complementary. But, selecting committees on the basis of complementary skills will need an analysis with more than one dimension. This is not the case here. Ratliff (2003), Ratliff and Saari (2014) studied committee selection by considering at least two dimensions. These authors came to the conclusion that selecting committees could be more complex than we might think. To be more precise, assume for example a hiring committee faced with 4 candidates (say, $a, b, c, d$ ) for two academic positions. Consider a member of the recruiting committee for whom candidates are ranked as $a b c d$ according to their research performances and $d c b a$ according to their teaching 
skills. If this voter is asked to rank pairs of candidates, he could put $(a, d)$ at the top of his ranking if he cares about complementary of skills; in the end, his ranking of committees is no longer lexicographic. Notice also that there is no guarantee that this voter behaves in this way. If he only cares about one skill (research or teaching) and if he is rational, we expect his ranking to be lexicographic in the sense that, if he prioritizes research skills, he will always rank $(a, b)$ at the top and $(c, d)$ at the bottom (he could say that he is indifferent between the other pairs). So, in such a context, many things can happen.

Let us now develop this example and present one extension rule under which the relationship between CCF and CCG may be totally broken: the preference for diversity. Given a voter's strict ranking on candidates, we will say that this voter has a preference for diversity over committees if he always prefers a committee made of his best and worse choices to those only made of his best (or worse) choices. This means that for a two-member committee, this voter will prefer a committee including his top and his bottom-ranked candidates to a committee made of his two top (bottom) candidates. To illustrate this, let us assume a voter with the ranking $a d b c$; his diversity-extended preferences will be: $(a, c) P^{*}(a, b) P^{*}(a, d) P^{*}(c, d) P^{*}(b, d) P^{*}(b, c)$. One can see that this ranking is quite different to what we get under the lexicographic approach.

So, with this kind of extension, we can end up with results contrasting with our previous theorems. The following examples will show that under the preference for diversity approach, anything can happen! For some voting profile, i) a committee can be both a CCG and a CCF; ii) there can be no CCG and no CCF; iii) there could be a CCG while there is no CCF; and iv) there could be a CCF while there is no CCG. The following examples illustrate all these situations.

Example 6. $(C C G=C C F) . \quad A=\{a, b, c, d\}$ and we have three voters with the following strict rankings:

$$
1: a P_{1} d P_{1} b P_{1} c, \quad 2: c P_{2} a P_{2} b P_{2} d \text { and } 3: c P_{3} d P_{3} b P_{3} a
$$

The diversity-extended preferences of these voters on two-member committees are as follows:

$$
\begin{array}{ccc}
1 & : & (a, c) P_{1}^{*}(a, b) P_{1}^{*}(a, d) P_{1}^{*}(c, d) P_{1}^{*}(b, d) P_{1}^{*}(b, c) \\
2 & : & (c, d) P_{2}^{*}(b, c) P_{2}^{*}(a, c) P_{2}^{*}(a, d) P_{2}^{*}(a, b) P_{2}^{*}(b, d) \\
3 & : & (a, c) P_{3}^{*}(b, c) P_{3}^{*}(c, d) P_{3}^{*}(a, d) P_{3}^{*}(b, d) P_{3}^{*}(a, b)
\end{array}
$$

The reader can check that committee $(a, c)$ is both the unique CCG and CCF. 
Example 7. $(C C G=\emptyset, C C F \neq \emptyset) . A=\{a, b, c, d\}$ and we have three voters with the following strict rankings:

$$
1: a P_{1} d P_{1} b P_{1} c, \quad 2: b P_{2} a P_{2} c P_{2} d \text { and } 3: c P_{3} d P_{3} b P_{3} a
$$

The diversity-extended preferences of these voters on two-member committees are as follows:

$$
\begin{array}{lcc}
1 & : & (a, c) P_{1}^{*}(a, b) P_{1}^{*}(a, d) P_{1}^{*}(c, d) P_{1}^{*}(b, d) P_{1}^{*}(b, c) \\
2 & : & (b, d) P_{2}^{*}(b, c) P_{2}^{*}(a, b) P_{2}^{*}(a, d) P_{2}^{*}(a, c) P_{2}^{*}(c, d) \\
3 & : & (a, c) P_{3}^{*}(b, c) P_{3}^{*}(c, d) P_{3}^{*}(a, d) P_{3}^{*}(b, d) P_{3}^{*}(a, b)
\end{array}
$$

One can check that for $g=2$, there is no CCG (due to the majority cycle among $a, b$ and d) while $(a, c)$ is the unique CCF by the diversity extension. This contrasts with what we get under the lexicographic extension.

Example 8. $(C C G=C C F=\emptyset) . A=\{a, b, c, d\}$ and we have three voters with the following strict rankings:

$$
1: a P_{1} d P_{1} b P_{1} c, \quad 2: b P_{2} a P_{2} c P_{2} d \text { and } 3: d P_{3} c P_{3} b P_{3} a
$$

The diversity-extended preferences of these voters on two-member committees are as follows:

$$
\begin{array}{lll}
1 & : & (a, c) P_{1}^{*}(a, b) P_{1}^{*}(a, d) P_{1}^{*}(c, d) P_{1}^{*}(b, d) P_{1}^{*}(b, c) \\
2 & : & (b, d) P_{2}^{*}(b, c) P_{2}^{*}(a, b) P_{2}^{*}(a, d) P_{2}^{*}(a, c) P_{2}^{*}(c, d) \\
3 & : & (a, d) P_{3}^{*}(b, d) P_{3}^{*}(c, d) P_{3}^{*}(a, c) P_{3}^{*}(b, c) P_{3}^{*}(a, b)
\end{array}
$$

Going from the diversity-extended preferences of these voters on two-member committees, the reader can check there is no CCG (due to the majority cycle among $a, b$ and $d$ ) and no CCF (due to the majority cycle among committees $(a, b),(a, d)$ and $(b, d))$.

The next example shows that for a given profile we can get a CCG that is not a $\mathrm{CCF}$ and vice-versa.

Example 9. (CCG $\neq C C F) . \quad A=\{a, b, c, d\}$ and we have five voters with the following strict rankings:

$1: a P_{1} b P_{1} d P_{1} c, 2: b P_{2} d P_{2} c P_{2} a, 3: c P_{3} b P_{3} d P_{3} a, 4: c P_{4} d P_{4} b P_{4} a$ and $5: d P_{5} a P_{5} b P_{5} c$

The diversity-extended preferences of these voters on two-member committees are as follows:

$$
\begin{array}{ccc}
1 & : & (a, c) P_{1}^{*}(a, d) P_{1}^{*}(a, b) P_{1}^{*}(b, c) P_{1}^{*}(b, d) P_{1}^{*}(c, d) \\
2 & : & (a, b) P_{2}^{*}(b, c) P_{2}^{*}(b, d) P_{2}^{*}(a, d) P_{2}^{*}(c, d) P_{2}^{*}(a, c) \\
3 & : & (a, c) P_{3}^{*}(c, d) P_{3}^{*}(b, c) P_{3}^{*}(a, b) P_{3}^{*}(b, d) P_{3}^{*}(a, d) \\
4 & : & (a, c) P_{4}^{*}(b, c) P_{4}^{*}(c, d) P_{4}^{*}(a, d) P_{4}^{*}(b, d) P_{4}^{*}(a, b) \\
5 & : & (c, d) P_{5}^{*}(b, d) P_{5}^{*}(a, d) P_{5}^{*}(a, c) P_{5}^{*}(a, b) P_{5}^{*}(b, c)
\end{array}
$$


With this profile, the reader can check that $C C G=(b, d)$ and $C C F=(a, c)$.

To conclude this discussion, we may consider that Axioms 1 and 2 of Kaymak and Sanver (2003) are too demanding, as well as our hypothesis of a "pure" leximin or a "pure" leximax extension or a mixed leximin-leximax. Even in these cases, the coincidence between the CCF and CCG is a tricky question since it relies upon Definition 6 and Property 4. But the examples we just invented shows that without a high degree of consistency between preferences on candidates and preferences on subsets, there is no reason to expect any relationships between both solution concepts.

\section{A natural way of getting preferences on candi- dates from those on committees}

When electing committees, the Candidate approach can be criticized if voters care about committees as a whole. Thus, the Committee approach seems to be the most natural departure point for committee elections and voters' preferences over committees are fundamental. According to Benoit and Kornhauser (1991, 1994, 1999), the Candidate approach may lead to the wrong combination of elected candidates and there is no guarantee that voters will act sincerely. According to these authors, the Committee approach provides a "natural" definition of sincerity ${ }^{8}$. Under the Committee approach, a sincere voter will prefer the committee for which she votes to any other committee for which she does not; and, she always prefer a member $x$ of a committee $C$ to a nonmember $y$ if and only if she prefers the committee $C$ to the committee $C \backslash\{x\} \cup\{y\}$. This defines a form of consistency between preferences on committees and those on alternatives that is called "Simple voting" by Benoit and Kornhauser (1991). We reappropriate this concept and adapt it to our context by defining an assumption on preferences for candidates rather than an assumption on voting behavior: the simple ranking.

Definition 9. (Simple ranking) Consider $x, y \in A$ and $Z \subseteq A$ such that $|Z|=g-1$. For $x, y \notin Z$, a voter $i$ has a simple ranking $x P_{i} y$ if and only if $\{x\} \cup Z R_{i}^{*}\{y\} \cup Z$ for all $Z$ and there is a subset $Z$ such that $\{x\} \cup Z P_{i}^{*}\{y\} \cup Z$.

The notion of simple ranking appears as a natural connection between preferences on committees and those on candidates when voters care about the committee as a whole. Notice that even though simple ranking seems "natural" and allows an obvious analysis of committee elections in terms of candidates, it may not be always

\footnotetext{
${ }^{8}$ For more on this, the reader can refer to Benoit and Kornhauser (1991, p.7).
} 
possible. Nonetheless, simple ranking is always possible if preferences on committees are separable $e^{9}$.

Definition 10. (Separable preferences on committees) For all $x, y \in A$ and $Z, Z^{\prime} \subseteq A$ such that $|Z|=\left|Z^{\prime}\right|=g-1$, preferences on committees are separable if for all voters $i,\{x\} \cup Z P_{i}^{*}\{y\} \cup Z$ implies $\{x\} \cup Z^{\prime} P_{i}^{*}\{y\} \cup Z^{\prime}$.

Example 10. Assume three voters with the following preferences on two-member committees on $A=\{a, b, c, d\}$.

$$
\begin{array}{ccc}
1 & : & (a, b) P_{1}^{*}(a, c) P_{1}^{*}(a, d) P_{1}^{*}(b, c) P_{1}^{*}(b, d) P_{1}^{*}(c, d) \\
2 & : & (a, b) P_{2}^{*}(a, c) P_{2}^{*}(b, c) P_{2}^{*}(a, d) P_{2}^{*}(b, d) P_{2}^{*}(c, d) \\
3 & : & (a, c) P_{3}^{*}(c, d) P_{3}^{*}(a, b) P_{3}^{*}(b, c) P_{3}^{*}(b, d) P_{3}^{*}(a, d)
\end{array}
$$

Since the preferences on committees for voters 1 and 2 are separable, their simple rankings will lead to the same ranking on candidates: abcd. There is no way to get the ranking on candidates for voter 3 since his preference is not separable; if we use the definition of simple ranking for this voter, we will get the following relationships: $a P_{3} b, b P_{3} a, a P_{3} c, c P_{3} a, b P_{3} d$ and $d P_{3} b$. We get some inconsistencies with voter 3.

According to Benoit and Kornhauser (1991, 1994, 1999), separability tends to enhance nonstrategic behavior since, if a voter has to cast $g$ votes, he will just vote for the top $g$ candidates of the ranking derived from his ranking on committees of size $g$. They admitted nonetheless that separability is restrictive. Given a set of candidates and the set of committees of size $g$, many voters can have different separable preferences on committees but their Simple rankings will lead to the same ranking on candidates (as in Example 10).

Proposition 5. If the preference $P^{*}$ on committees is separable, then under simple ranking the preference $P$ on $A$ is unique. Moreover, $P^{*}$ satisfies the Strict (Extended) Independence (Properties 3 and 4).

Notice that if preferences are separable, Simple ranking implies that Equation 3.1 holds whenever $X$ and $Y$ satisfy the conditions in Proposition 1; it follows that Propositions 3 and 4 are also met when preferences are separable. Then, we can state the following.

Theorem 4. Consider a preference profile with $m \geq 3$ candidates such that voters' preferences on committees of size $g$ are separable. If there is a CCF this implies that under simple ranking there is also a CCG and it coincides with the CCF. If the simple ranking leads to a situation with no $C C G$, this implies that there is no $C C F$.

\footnotetext{
${ }^{9}$ This also apply for the Simple voting as noticed by Benoit and Kornhauser $(1991,1994,1999)$.
} 
The proof of Theorem 4 follows the scheme of that of Theorems 2 and 3. Notice that, given a set of candidates and the set of committees of size $g$, the set of the separable preferences on committees that lead to a given strict ranking on candidates always contains both the Leximax and Leximin rankings associated to this strict ranking on candidates. A patent case is for $m=4$ and $g=2$ where the set of the separable preferences associated to a given ranking on candidates is only made of the associated Leximax and Leximin rankings. Then, we cannot exclude that one can end with preferences on committees made of a mixture of Leximax and Leximin rankings and Remark 2 holds.

\section{Conclusion}

Given the possible inconsistencies pointed out by Coelho (2004) on the debate between Dodgson (1884, 1885a,b) and the Society for Proportional Representation concerning committee elections, the aim of this paper was to revisit the relationship that could exist between two famous solution concepts: the Condorcet Committee à la Gerhlein (for the Candidate approach), and the Condorcet Committee à la Fishburn (for the Subset approach). Kaymak and Sanver (2003) indeed proved that a committee is a CCF if and only if there exist extensions of preferences satisfying certain conditions that ensure it will also be undominated at any preference profile. Their result was presented as a positive one, as a CCF seems to have a good chance of existing most of the time. However, a weakness of their result comes from the fact that, to obtain the equivalence between an undominated committee (which is also a CCG at some instances) and a CCF, different extensions within their class may have to be used at different profiles. The objective of our paper was to revisit their result when all the voters systematically use the same extension at each profile. In this paper, we focused in particular on the Leximax and the Leximin extensions, specific cases contained in the class of extensions proposed by Kaymak and Sanver (2003). We showed that the non-existence of the CCG in a profile also implies the non-existence of the CCF by lexicographic extension. However, for some profiles, a CCG may exist while that is not the case for the CCF. Finally, we discussed the natural way of deriving preferences on candidates from those on committees. This approach also has a limit, as it strongly depends on the hypothesis of separability.

Our results may not hold if the assumption of the lexicographic extension is dropped. It will be interesting to know what happens under the other extension methods known in the social choice literature. We could also investigate cases where some restrictions (gender, quota, skills etc) are made on the composition of the committees as in Ratliff and Saari (2014). We expect that, by taking such restrictions 
into account, this may induce other types of preferences or ways of deriving preferences on committees that could reconcile the candidate approach and the committee approach of the selection of committees.

Furthermore, the computational social choice community has recently explored many routes to analyzing committee elections. Just to give a few examples, these researchers have explored the way to model preferences in complex environments (Lang and Xia (2016)), new dominance concepts that go beyond the majority rule (Elkind et al. (2015)) or the complexity of deriving these new solutions (Darmann (2013); Aziz et al. (2016)). Also, the axiomatic approach has been successfully used in order to analyze the committee solutions based upon scoring rules (see for example Elkind et al. (2017)).

Both in the candidate approach (with lexicographic extension) and the committee approach (with separable preferences), we have assumed that voters are sincere. The strategic aspect is not tackled in this paper. This question was also absent in the original paper of Kaymak and Sanver (2003). We could envision this aspect by examining the problem as a game, the preferences being set on candidates and the space of strategies being the set of all the possible preferences on subsets and vice-versa. This would raise the following question: which voting rule is then implementable? We felt that a deep examination of this question is beyond the scope of this paper.

Acknowledgements. We are grateful to Bernard Grofman, Hans Peters, Ashley Piggins, Claudio Zoli and to two anonymous reviewers for all their valuable comments. We would like to thank all the participants of the Voting sessions at the 2013 Annual Meeting of the Public Choice Society (New Orleans), at the 2013 Annual Meeting of the Association for Public Economic Theory (Lisbon) and at the 2013 joint 62nd AFSE Meeting-12th Journées LAGV (Aix-en-Provence). Thanks also to Reiko Gotoh and the members of the Institute of Economic Research-Hitotsubashi University (Japan) for their remarks and suggestions. Vincent Merlin acknowledges the support from the project ANR-14-CE24-0007-01 CoCoRICo-CoDec.

\section{References}

Arrow K.J, Hurwicz L (1972) An optimality criterion for decision-making under ignorance. In: Carter, C.F. and Ford, J.L. (eds.), Uncertainty and Expectations in Economics: Essays in Honour of G.L.S. Shackle, Basil Blackwell, Oxford. 
Aziz H, Lang J, Monnot J. (2016). Computing Pareto Optimal Committees. IJCAI 2016: 60-66.

Barberà S, Barrett C.R, Pattanaik P.K. (1984). On some axioms for ranking sets of alternatives. Journal of Economic Theory 33: 301-308.

Barberà S, Bossert W, Pattanaik P.K (2001) Ordering Sets of Objects. In: Salvador Barberà, P.J. Hammond and C. Seidl(eds.), Handbook of Utility Theory. Vol.2, Ch.17. Kluwer Academic Publishers, Dordrecht-Boston.

Barberà S, Coelho D (2008) How to choose a non-controversial list with k names. Social Choice and Welfare 31: 79-96.

Benoit J.P, Kornhauser L.A (1991)Voting Simply in the Election of Assemblies. Technical report 91-32, C.V. Starr Center for Applied Economics, New York University.

Benoit J.P, Kornhauser L.A (1994) Social choice in a representative democracy. American Political Science Review 88: 185-192.

Benoit J.P, Kornhauser L.A (1999) On the separability of assembly preferences. Social Choice and Welfare 16: 429-439.

Black D (1958) The Theory of Committees and Elections Cambridge University Press, Cambridge.

Coelho D (2004) Understanding, evaluating and selecting voting rules through games and axioms. Phd Thesis, Universitat Autonoma de Barcelona. Available at http: //ddd.uab.es/record/36576? ln=en.

Marquis de Condorcet (1785) Essai sur l'Application de l'Analyse à la Probabilité des Décisions Rendues à la Pluralité des Voix. Paris.

Darmann A. (2013) How hard is it to tell which is a Condorcet committee? Mathematical Social Sciences 66(3): 282-292.

Dodgson C.L (1876) A Method of Taking Votes on More than Two Issues. Clarendon Press, Oxford.

Dodgson C.L (1884) The principles of parliamentary representation. Harrison and Sons Publisher, London.

Dodgson C.L (1885a) The principles of parliamentary representation: Postscript to Supplement, E. Baxter Publisher, Oxford. 
Dodgson C.L (1885b) The principles of parliamentary representation: Supplement, E. Baxter Publisher, Oxford.

Elkind E., Faliszewski P., Skowron P. Slinko A. (2017) Properties of Multiwinner Scoring Rules. Social Choice and Welfare 48: 599-632.

Elkind E., Lang J., Saffidine A. (2015) Condorcet Winning Sets. Social Choice and Welfare 44(3): 493-517.

Fishburn P.C (1981) An analysis of simple voting systems for electing committees. SIAM Journal on Applied Mathematics 41: 499-502.

Gehrlein W.V (2006) Condorcet's Paradox, Springer Publishing.

Gehrlein W.V (1985) The Condorcet criterion and committee selection. Mathematical Social Sciences 10: 199-209.

Good I.J (1971) A note on Condorcet sets. Public Choice 10(1): 97-101.

Hill I.D (1988) Some aspects of elections: To fill one seat or many. Journal of the Royal Statistical Society 151: 243-275.

Kaymak B., Sanver M.R (2003) Sets of alternatives as Condorcet winners. Social Choice and Welfare 20: 477-494.

Kamwa E. (2017) On stable rules for selecting committees. Journal of Mathematical Economics 70: 36-44.

Kamwa E. (2017) Stable Rules for Electing Committees and Divergence on Outcomes. Group Decision and Negotiation 26 (3): 547-564.

Lang J, Xia L. (2016) Voting in combinatorial domains. In: F. Brandt, V. Conitzer, U. Endriss, J. Lang and A.D. Procaccia (eds.), Handbook of Computational Social Choice, Ch 9. Cambridge University Press.

McLean I, Urken A. (1995) Classics of Social Choice. Michigan University Press.

Nitzan S., Pattanaik P.K.(1984) Median-based extensions of an ordering over a set to the power set: an axiomatic characterization. Journal of Economic Theory 34: 252-261.

Packard D.J (1979) Preference relations. Journal of Mathematical Psychology 19: 295-306. 
Pattanaik P.K, Peleg B (1984) An axiomatic characterization of the lexicographic maximin extension of an ordering over a set to the power set. Social Choice and Welfare 1: 113-122.

Pérez J, Jimeno J.L, García E (2012) No Show Paradox in Condorcet k-voting Procedures. Group Decision and Negotiation 21(3): 291-303.

Ratliff T.C (2003) Some startling inconsistencies when electing committees. Social Choice and Welfare 21:433-454.

Ratliff T.C, D.G. Saari (2014) Complexities of electing diverse committees. Social Choice and Welfare 43:55-71. 